\title{
DIADOLAB: bilan court et objectif de la dysarthrie dans le cadre de la prise en charge orthophonique
}

Auteurs:

- Etienne SICARD, Professeur INSA de Toulouse, Directeur de Recherches ERU 46 LURCO/Unadreo, chercheur associé IRIT, etienne.sicard@insa-toulouse.fr

- Anne SICARD, Orthophoniste, chercheuse ERU 46 LURCO/Unadreo, Formatrice voix et parole, anne.sicard2@orange.fr

\section{Résumé:}

Nous présentons dans cet article un ensemble d'outils originaux développés pour l'analyse objective de la parole dysarthrique dans le cadre de la prise en charge orthophonique. Le bilan rapide de la dysarthrie repose deux épreuves principales : une courte phrase et une répétition de /PaTaKa/. La phrase a été choisie pour couvrir en un minimum de phonèmes, les extrêmes vocaliques, et consonantiques, couvrir plus de $80 \%$ des processus de parole, avec une phonotactique proche de la langue française ainsi que l'inclusion des phonèmes d'occurrence la plus élevée. L'analyse de la phrase permet, par segmentation assistée, d'établir un score d'articulation, puis d'extraire les informations de prosodie, notamment le contour intonatif, les accents et d'éventuelles pauses inappropriées. L'analyse de la diadococinésie permet quant à elle d'extraire quatre indicateurs en lien direct avec les effets de la dysarthrie : atonie des plosives, ralentissement du débit, irrégularité du débit, et irrégularité de la puissance. Le bilan est complété par l'analyse d'une voyelle tenue et la capacité à reproduire des praxies, et optionnellement l'analyse prosodique d'une phrase. Les outils ont été implémentés dans la plateforme DIADOLAB, qui servent conduire l'ensemble des étapes du bilan. Nous décrivons en quoi le bilan d'évolution proposé permet d'objectiver les progrès du patient bénéficiant d'une prise en charge orthophonique, autant sur les aspects intelligibilité que fluence.

\section{Mots clé :}

Dysarthrie, bilan de la parole, analyse objective, diadococinésie 


\section{Introduction}

L'analyse de la parole dysarthrique a fait l'objet d'un nombre conséquent de recherches, notamment pour évaluer la gravité des troubles de la parole, mais aussi permettre le diagnostic différentiel précoce, ou encore faciliter l'accompagnement des patients dans le cadre de la prise en charge orthophonique. L'évaluation de la dysarthrie se fait aujourd'hui en pratique orthophonique soit à l'aide de logiciels tels que VOCALAB [Sicard 2013], peu adapté à la parole, soit plus généralement à l'aide de batteries d'évaluation dédiées telles que la BECD [Auzou 2006], incluant un grand nombre d'épreuves mais ne bénéficiant pas d'outil de mesures objectives dédié.

Dans cet article, nous proposons un bilan de la dysarthrie rapide, associé à logiciel support qui facilite et automatise un grand nombre de tâches telles que la cotation, la comparaison aux valeurs référentielles en fonction de l'âge et la rédaction du compte-rendu. L'originalité de l'approche est de limiter le protocole à un ensemble très restreint de d'épreuves, intégrées dans une interface conviviale et un outil de génération de fiche bilan efficace.

Après avoir donné quelques éléments généraux sur les troubles de la parole, les dysarthries et la parole Parkinsonienne, nous décrivons notre protocole de bilan, en approfondissant l'analyse d'une phrase courte, et l'extraction d'indicateurs sur une épreuve de diadococinésie, que nous illustrons avec certains exemples de parole normale, Maladie de Parkinson PD) et Multiple System Atrophy (MSA). La compilation des différents indicateurs nous donne des éléments d'intelligibilité et de fluence que nous nuançons avec l'analyse subjective du clinicien. Enfin, au travers de différentes analyses de corpus et de projets de recherche, nous donnons quelques caractéristiques clé de la parole Parkinsonienne, dans le but d'une aide à la classification de la sévérité de la dysarthrie.

\section{Classification des troubles de la parole}

Nous décrivons tout d'abord la dysarthrie dans le contexte général des troubles moteurs de la parole, puis abordons plus spécifiquement la Maladie de Parkinson (Idiomatic Parkinson's Disease - IPD) ainsi que deux syndromes spécifiques, Multiple System Atrophy (MSA) et Progressive Supranuclear Palsy (PSP). Nous analysons ensuite quelques traits distinctifs de la parole Parkinsonienne avant de décrire les principaux éléments du bilan rapide de la parole.

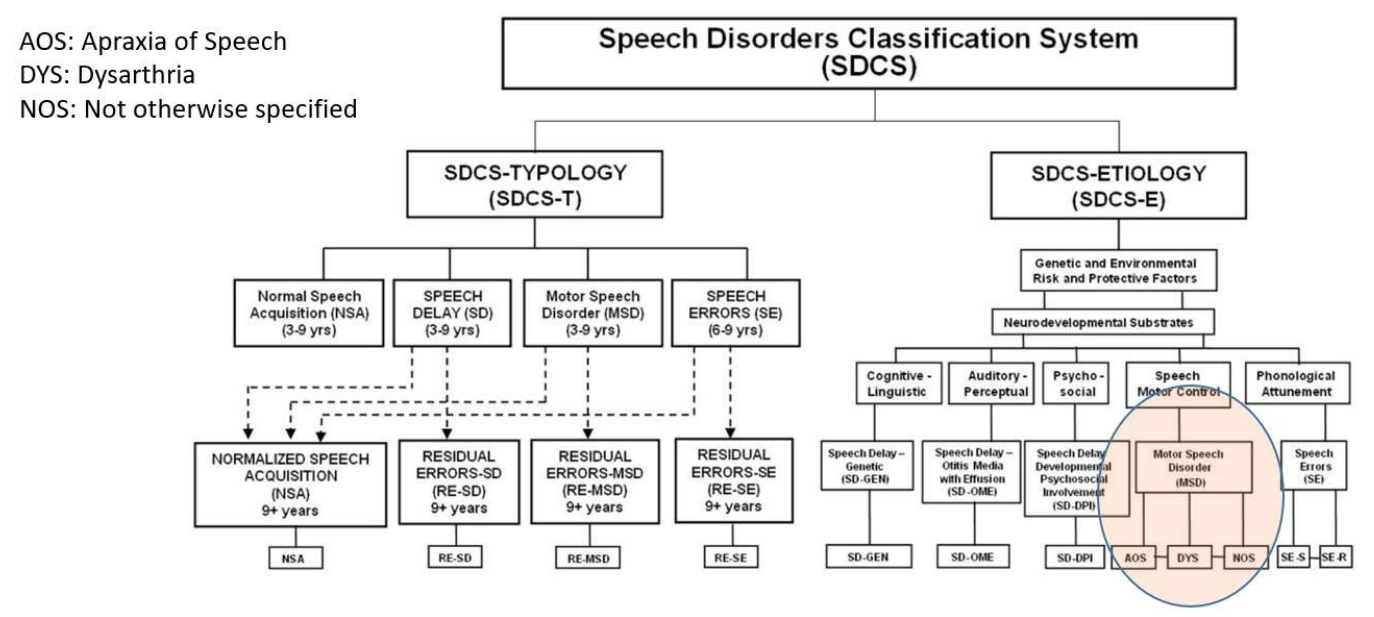

Figure 1 : classification des troubles de la parole, avec la rubrique dysarthrie (DYS) qui apparaît dans la sousclasse Motor Speech Disorders (MSD) 
Parmi tous les troubles, les désordres moteurs de la parole (Motor Speech Disorders) concernent plus particulièrement les dysarthries. La classification publiée dans [Shriberg 2010] est reportée Figure 1. La classification de [Tranchant 2017] considère l'Atrophie Multi Systématisée (MSA) et la Paralysie Supra-nucléaire Progressive (PSP) comme des syndromes dégénératifs sporadiques, tandis que la Maladie de Wilson est considérée comme d'origine métabolique héréditaires.

\section{Types de dysarthries}

Aux syndromes Parkinsoniens sont rattachés de façon plus ou moins simple, différents types de dysarthries. La Dysarthrie Hypokinétique (HOD) est souvent rattachée à la Maladie de Parkinson Idiomatique (IPD), mais il est plus difficile de rattacher les différents syndromes Parkinsoniens à un seul type de dysarthrie. On parle alors de composante ataxique (AD), hypokinétique (HOD), hyperkinétique (HYD), spastique (SD) voir mixte (MD) [Fonville 2008][Auzou 2009].

Les Clusters de Darley [Darley 1969] traduits par Auzou [Auzou 2009] sont une tentative d'illustrer les traits caractéristiques de la voix et de la parole en regroupant certaines caractéristiques sous forme de groupes, listés ci-dessous. Le trois dysarthries illustrées figure 3 (Spastique, Ataxique et Hypokinétique) montrent une prédominance de certains clusters. Au total, sept clusters sont identifiés :

1. Imprécision articulatoire

2. Excès prosodique

3. Insuffisance prosodique

4. Incompétence résonantielle et articulatoire

5. Sténose phonatoire

6. Incompétence phonatoire

7. Incompétence résonance

- Spastique

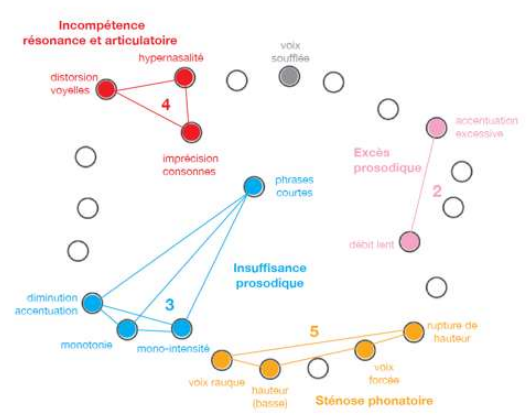

- Ataxique

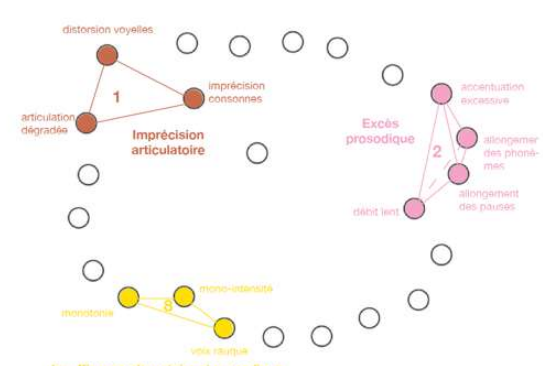

- Hypokinétique

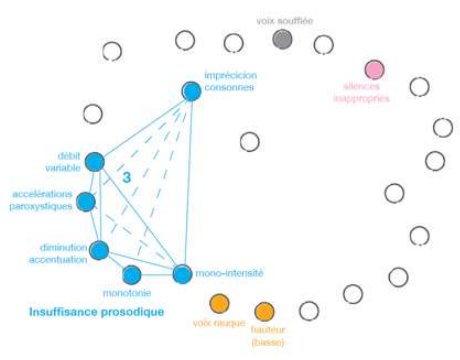

Figure 2: Clusters de Darley traduits par Auzou [2009], illustration avec 3 types de dysarthrie: spastique, ataxique et hypokinétique

\section{PD, MSA et PSP}

Nous reportons au tableau 1 quelques caractéristiques comparatives de la Maladie de Parkinson et de deux syndromes: MSA et PSP [Miller 2017]. II est possible de relier la maladie avec un type de dysarthrie prédominant, notamment pour PD que l'on associe à la dysarthrie hypokinétique. Différentes échelles sont utilisées comme UPDRS et NNIPPS [Payan 2011]. 


\begin{tabular}{|c|c|c|c|c|c|c|}
\hline Disease & Acronym & Prevalence & $\begin{array}{l}\text { Mean age } \\
\text { of onset } \\
\text { (years old) }\end{array}$ & Rating scale & $\begin{array}{l}\text { Mean } \\
\text { survival } \\
\text { (years) }\end{array}$ & Type of dysarthria \\
\hline $\begin{array}{l}\text { Parkinson } \\
\text { Disease }\end{array}$ & PD & $\begin{array}{l}1 \% \text { of people } \\
\text { over } 60 \text { y.old. }\end{array}$ & 65 & UPDRS & $>10$ & hypokinetic \\
\hline $\begin{array}{l}\text { Multiple system } \\
\text { atrophy }\end{array}$ & MSA & $5-8 / 100,000$ & 58 & NNIPPS & $5-10$ & $\begin{array}{l}\text { predominantly } \\
\text { hypokinetic and spastic } \\
\text { characteristics }\end{array}$ \\
\hline $\begin{array}{l}\text { Progressive } \\
\text { supranuclear } \\
\text { palsy }\end{array}$ & PSP & $4-6 / 100,000$ & $63-65$ & NNIPPS & $5-10$ & $\begin{array}{l}\text { more prominent ataxic } \\
\text { changes alongside } \\
\text { hypokinetic speech }\end{array}$ \\
\hline
\end{tabular}

Table 1 : Comparaison entre PD, MSA et PSP

\section{Analyse de la parole Parkinsonienne}

Nous passons en revue ci-après un ensemble de paramètres souvent étudiés dans le cadre de la dysarthrie Parkinsonienne.

\section{Paramètres généraux}

La maladie de Parkinson se traduit généralement par des mouvements plus lents de plus faible amplitude, des capacités phonatoires altérées, notamment une voix plus faible et monotone, ce qui tend à diminuer l'intelligibilité du patient. Le principe de la prise en charge LSVT'M proposée par FOX et Ramig [Fox 2012] (Figure 3) se focalise sur le travail de la puissance de la voix, l'augmentation de l'amplitude des mouvements, de l'intonation afin d'améliorer l'intelligibilité et préserver les capacités de communication.
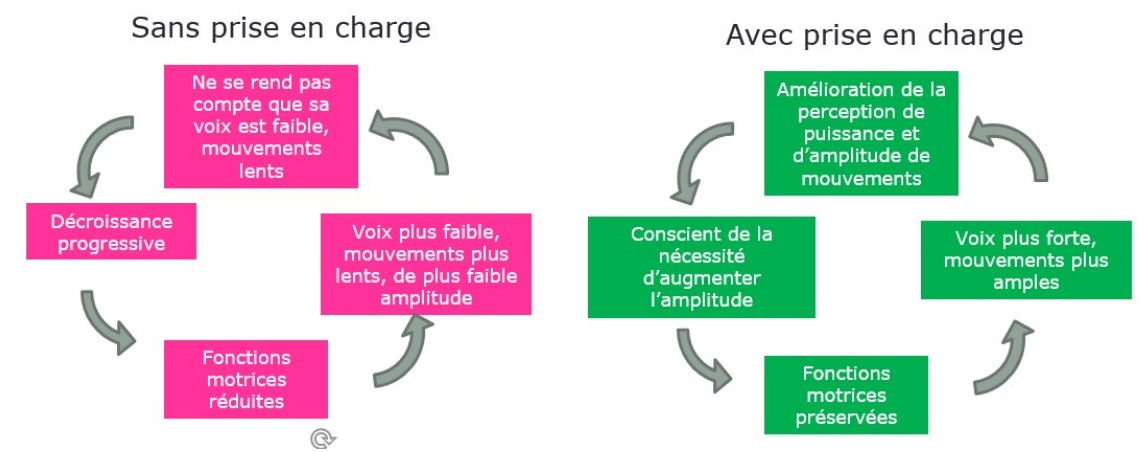

Figure 3 : Principe de base de la méthode LSVT тм [Fox 2012]

A l'échelle des phonèmes, l'erreur articulatoire la plus typique de la dysarthrie parkinsonienne est la réalisation des consonnes occlusives comme des fricatives [Sarr 2013]. Dans certaines dysarthries, les consonnes sourdes se voisent, par exemple à cause de la rigidité du larynx [Tykalova 2017]. Parmi d'autres effets, on peut citer une perte de contrôle du voice onset time (VOT), c'est-à-dire du temps qui s'écoule entre le relâchement de la consonne et le début du voisement, à cause de défauts de coordination entre le larynx et les organes articulatoires (Illustration à la figure 3 ). Entre groupe PD et $\mathrm{HC}$, on observe principalement une augmentation du VOT sur les plosives /p,t,k/ (Fig. 4) ainsi que la réduction du voisement sur $/ \mathrm{b}, \mathrm{d}, \mathrm{g} /$. Les dysarthries les plus sévères sont celles en lien avec PSP et MSA, ce qui confirme des résultats similaires tels que [Auzou 2000]. 

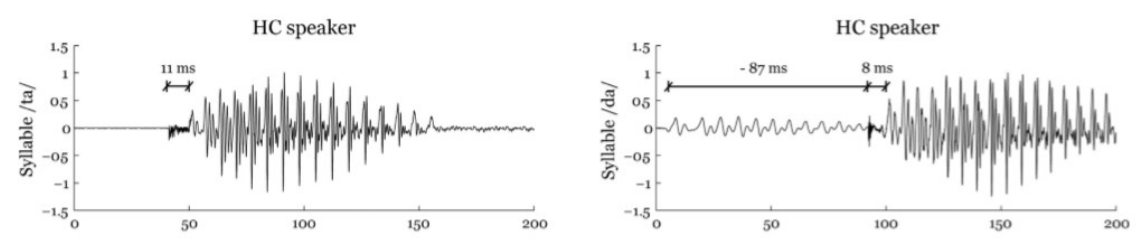

PD patient
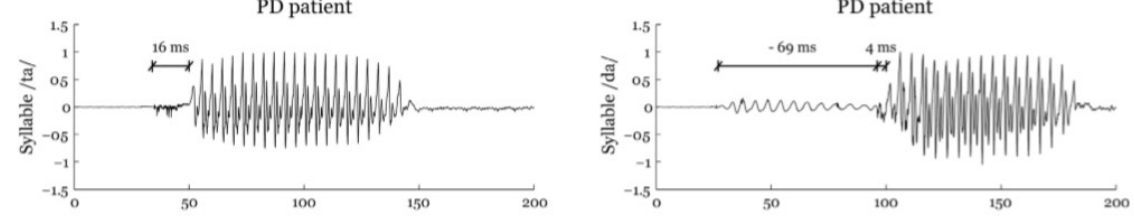

Figure 4 : Illustration des variations de VOT sur /ta/ et /da/ en parole normale (HC) et Maladie de Parkinson (PD) par [Tykalova 2017].

Lorsque les articulateurs perdent en force et en précision l'occlusive peut perdre son mode et son lieu d'articulation (Locco 2005, Tykalova 2017);

1. Spirantisation: I'occlusive peut devenir fricative par changement du lieu d'articulation
a. le $/ \mathrm{p} /$ devient $/ \mathrm{f} /$,
b. le $/ \mathrm{t} / \mathrm{devient} / \mathrm{s} /$
c. le $/ \mathrm{k} /$ devient /ch/

2. Consonne voisée: ajout d'une constriction

a. le $/ d /$ devient $/ z /$,

b. le /b/devient /v/,

3. Consonne voisée: suppression du voisement (MSA principalement)
a. le $/ \mathrm{b} /$ devient $/ \mathrm{p} /$,
b. le $/ d /$ devient $/ \mathrm{t} /$,
c. le $/ \mathrm{g} /$ devient $/ \mathrm{k} /$

L'analyse de différentes caractéristiques de la parole altérée et pathologique a été conduite par Rusz [2015] en rapprochant les traits de la parole aux types de dysarthries (Tableau 3). Par exemple, la parole monotone (Monopitch) est rattachée à un trait de la dysarthrie hypokinétique, que l'on peut aussi relier à l'item « Monotonie » du cluster de Darley (Figure 2). 


\section{No.}

Deviant speech dimension

\section{PSP}

MSA

PD

\begin{tabular}{|c|c|c|c|c|}
\hline & Airflow insufficiency & Common (42 \%) & Common (31 \%) & Common (27 \%) \\
\hline & Harsh voice & Abundant (75\%) & Frequent (69 \%) & Occasional (13\%) \\
\hline & Rapid AMR & Occasional (25\%) & Common (31 \%) & Occasional (13\%) \\
\hline & Inappropriate silences & Abundant (83\%) & Frequent (69 \%) & Common (27 \%) \\
\hline & Reduced loudness & Occasional (25 \%) & Occasional (23 \%) & Occasional (13\%) \\
\hline & Monopitch & Frequent (50\%) & Common (31\%) & Frequent (53 \%) \\
\hline & Imprecise vowels & Abundant (75\%) & Frequent (62\%) & Common (33 \%) \\
\hline & Dysfluency & Frequent (58 \%) & Occasional (15\%) & Occasional (13\%) \\
\hline \multicolumn{5}{|l|}{ Spastic } \\
\hline & Strained-strangled voice & Common (42\%) & Frequent (62 \%) & Rare (0\%) \\
\hline & Slow AMR & Frequent (50\%) & Frequent (54 \%) & Rare (0\%) \\
\hline & Slow rate & Common (42 \%) & Occasional (23 \%) & Rare $(0 \%)$ \\
\hline \multicolumn{5}{|l|}{ Ataxic } \\
\hline & Excess pitch fluctuations & Common (33 \%) & Frequent (69 \%) & Rare (0\%) \\
\hline & Vocal tremor & Common (33 \%) & Frequent (54 \%) & Rare (0\%) \\
\hline & Irregular AMR & Common (33 \%) & Common (31 \%) & Rare $(0 \%)$ \\
\hline & Prolonged phonemes & Occasional (25 \%) & Frequent (54 \%) & Rare $(0 \%)$ \\
\hline
\end{tabular}

Table 3 : Comparaison entre PD, MSA et PSP pour différents traits dysarthriques [Rusz 2015]

Débit de la parole

Les patients atteints de la MP présentent une certaine diminution du débit de parole, comme illustré figure 5 [Martinez-Sanchez, 2016]. Ce ralentissement est le résultat des troubles du mouvement qui affectent la physiologie des systèmes de production de la parole. On note un ralentissement du débit beaucoup plus conséquent pour des syndromes tels que PSP et MSA.

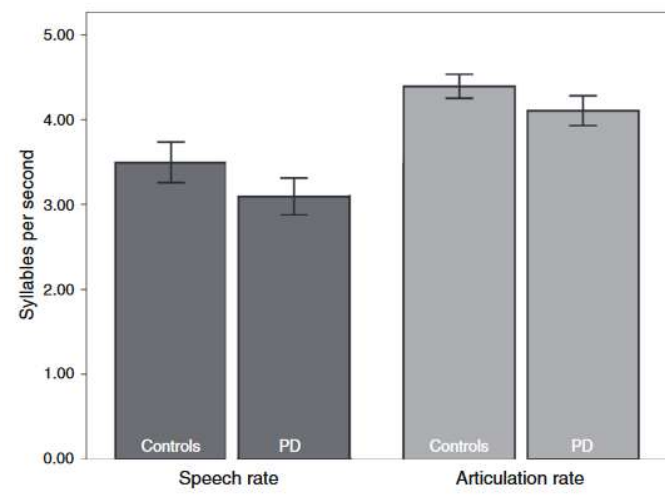

Figure 5 : Débit de parole (à gauche) et d'articulation (à droite), HC vs PD [Martínez-Sanchez, 2016] 


\section{Triangle vocalique}

Certains auteurs tels que [Orozco-Arroyave 2014] ou [Roland 2017] ont analysé les caractéristiques du triangle vocalique, dans une représentation de l'espace des formants F1 et F2. Il est soit constaté une diminution de l'aire du triangle (Fig. 6), soit une variabilité importante de cette aire pour les patients PD.

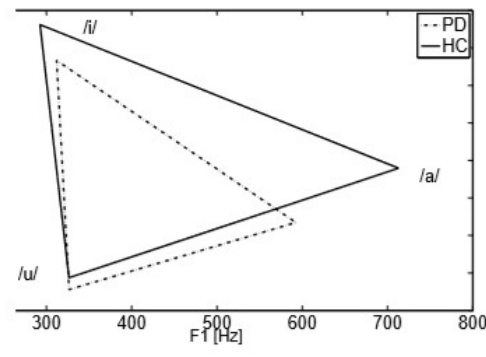

(a) $t V S A$

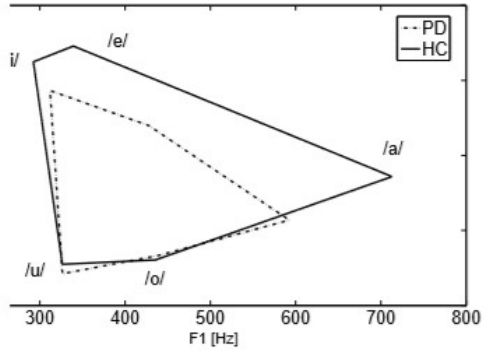

(b) aPenta

Figure 6: Modification du triangle vocalique /aiou/ (gauche) ou laiouoel, HC vs PD [Orozco-Arroyave 2014]

En lien avec les voyelles, on peut aussi citer les travaux de [Duez 2006] sur l'allongement de l'ordre de $10 \%$ de la durée de voyelles et la réduction de la durée des consonnes du même ordre, entre parole Parkinsonienne et parole normale.

\section{Hypernasalité}

L'hypernasalité dans la dysarthrie a été notamment étudiée par [Novotny 2016] en ciblant l'analyse acoustique sur la voyelle / $\mathrm{i}$ / et plus particulièrement la bande autour de $1 \mathrm{kHz}$. Dans le cadre d'une analyse perceptive (Groupe $\mathrm{C}$ de la figure 7), une hypernasalité légère à modérée a été constatée dans $65 \%$ des cas de PD, $89 \%$ de la maladie de Huntington et $22 \%$ des témoins (HC). Sur la base d'analyses acoustiques ciblant la bande $1 \mathrm{KHz}$, des tendances similaires ont été observées.

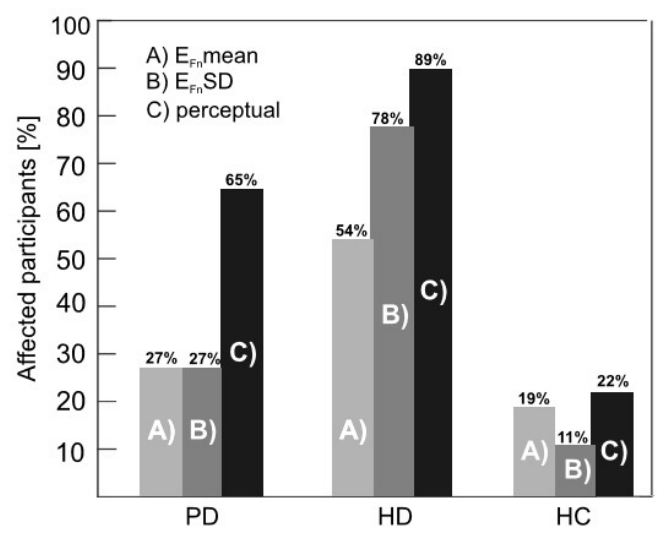

Figure 7 : Hypernasalité dans le cadre de la Maladie de Parkinson et de Huntington [Novotny 2016]

\section{Diadococinésie}

La diadococinésie (Diadochokinetic - DDK) est une épreuve de répétitions de syllabes, la plus connue étant la répétition de / $\mathrm{PaTaKa} /$. Elle permet de mesurer le débit d'articulation (Alternating motion rate - AMR). L'analyse porte en général sur 5 à 10 secondes de production. Certaines épreuves consistent à répéter la même syllabe comme /pa/pa/pa.. ou /ta/ta/ta.., on parle alors de Sequential motion rate (SMR). Dans le cadre de ce type d'épreuve, on cherche à mesurer la capacité du sujet à répéter une combinaison consonne-voyelle $(C-V)$ avec des lieux d'articulation différents : bilabial, alvéolaire et vélaire. Concernant la parole dysarthrique, les paramètres les plus étudiés concernent : 
- Le débit syllabique (syllabe / seconde): lent, normal, rapide

- Les variations de débit : régulières / irrégulières, accélérant, ralentissant [Rusz 2015]

- Les variations de puissance de phonation : décroissance rapide dans le cas de la dysarthrie [Rusz 2011]

- La qualité des phonèmes : plosives énergétiques, taux de constriction, voisement

Une grande variabilité des protocoles existe en France selon les centres référents [Jan 2007] : débit et intensités sur " papapa ", sur /pa/, /ta/ \& /ka/, sur /Ba/, /Cla/, /Tra/, ou sur une phrase telle que " Papa ne m'a pas parlé de beau-papa ". Le protocole MonPaGe [Fougeron 2016] inclut aussi différentes épreuves dont la mesure du débit sur /Bababa/ (Consone Voyelle CV), /Dédédé/ avec une consonne dentale antérieure et une voyelle facilitatrice, /GoGoGo/avec une consonne vélaire postérieure et une voyelle facilitatrice, enfin les clusters CCV avec/ClaClaCla/,/TraTraTra/, ainsi que /Badégo/ et/ClaTra/.

Le choix de /PaTaKa/ dans notre proposition de bilan est guidé par différents arguments : la voyelle /a/ est universelle, la plus ouverte, riche en harmoniques et la plus énergétique, constitue une extrême $\mathrm{du}$ triangle vocalique, les plosives $/ \mathrm{p}, \mathrm{t}, \mathrm{k} /$ sont énergétiques, requièrent une bonne tonicité et nécessitent un déplacement important de la langue et un mouvement des lèvres. Ces enchainements non facilitateurs permettent d'évaluer les capacités de programmation motrices et de contrôle automatico-volontaire. Les calculs sont facilités par la dynamique du signal. On peut aussi mettre à profit de très nombreuses données publiées sur /pataka/, notamment en lien avec la dysarthrie, pour différentes populations $(H, F, E)$, et l'on note globalement très peu de différence selon le genre.

Le choix d'un protocole focalisé sur /PaTaKa/ donc sur la précision d'articulation et la puissance, plutôt que sur le débit maximum, permet d'éviter un biais en lien avec la recherche de rapidité d'exécution. Les résultats de la méta-analyse des données scientifiques sur le débit de / $\mathrm{PaTaKa/} \mathrm{sont} \mathrm{reportés} \mathrm{figure}$ 8 en fonction de l'âge du groupe témoin étudié.

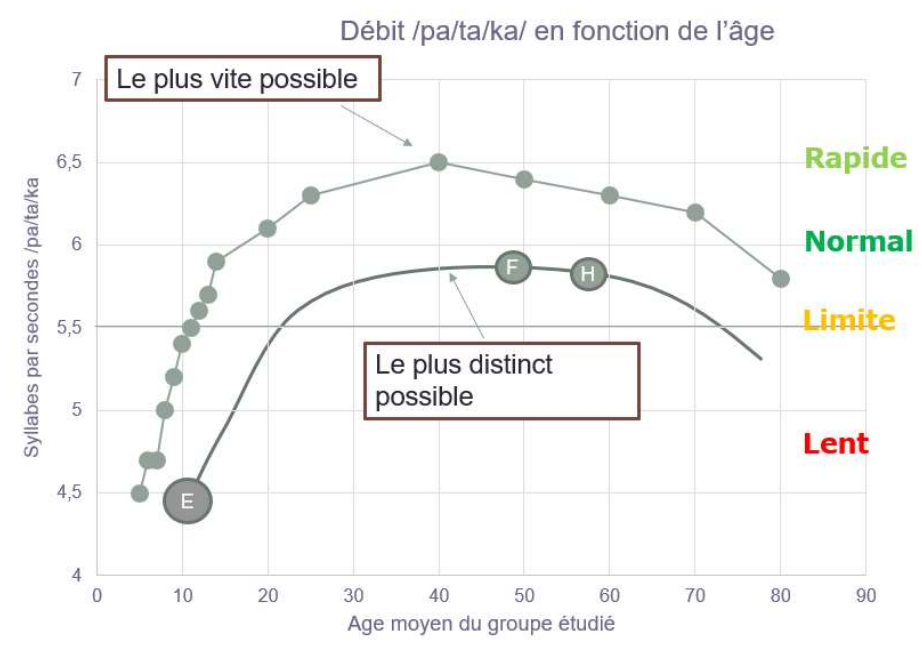

Figure 8: Méta-analyse des publications sur /PaTaKa/ en fonction de l'âge, chaque point correspondant à la moyenne obtenue pour une publication donnée.

La tendance observée par [Rusz 2015] est au ralentissement du débit (AMR) pour environ 50\% des patients MSA et PSP, mais très peu concernant PD (Tableau 3). Ce paramètre peut donc être considéré comme différentiateur entre HC-PD et MSA-PSP. La différence entre DDK de témoin et de patient dysarthrique (Ici MSA) est illustrée Figure 9. On note un débit significativement plus bas que la norme (3.5 phonèmes/seconde), une irrégularité marquée (absence d'un pic net dans le spectre de puissance) et une décroissance de la puissance au cours de la production. 

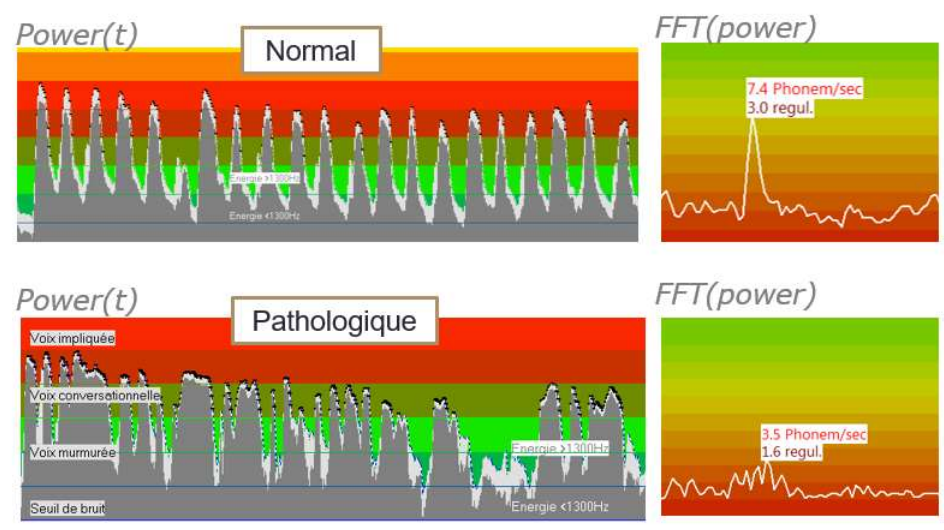

Figure 9 : Répétitions de /PaTaKa/ d'un sujet contrôle et d'un patient atteint de dysarthrie (MSA)

\section{Protocole d'évaluation de la dysarthrie}

\section{Protocoles existants}

Différents protocoles d'évaluation de la dysarthrie existent, sur la base des travaux de [Kent 1979] à destination des orthophonistes. Le plus utilisé en France est sans doute la batterie d'évaluation clinique de la dysarthrie BECD [Auzou 2006-2019]. En particulier, le Test Phonétique d'Intelligibilité (TPI) permet de mesurer la sévérité de la dysarthrie d'après la lecture par le patient de 52 items parmi 4 propositions, que l'évaluateur doit reconnaître, la lecture d'un texte avec caractérisation des erreurs, une épreuve de répétition de mots simples et complexes.

Le protocole MonPaGe [Fougeron 2016] est un outil informatisé d'évaluation de la parole pathologique en langue française. Il est composé de divers modules incluant notamment l'analyse de voyelles tenues, de pseudo-mots, de diadococinésie et de prosodie. Ce protocole a notamment donné lieu à des travaux de recherche en lien avec l'Ataxie de Friedrich, mais n'est pas encore déployé en pratique libérale.

On peut aussi citer le test d'intelligibilité Frenchay Dysarthria Assessment [Enderby 2008] dont la traduction en langue Française a fait l'objet du mémoire de [Blanc 2014]. Dans le cadre d'un projet plus général de l'évaluation de l'intelligibilité, [Ghio 2016] suggère le décodage acoustico-phonétique d'une série de 52 logatomes.

Bien que conçu pour l'analyse de la voix, VOCALAB [Sicard 2014] permet d'analyser différents traits caractéristiques de la voix de patients dysarthriques [Jan, 2007] dans le cadre de la prise en charge et du suivi orthophonique. Les paramètres souvent analysés sont en lien avec la phonation :

- L'étendue vocale (sirènes ascendante et descendante)

- Les indicateurs d'altération du /a:/ tenu impliqué

- $\quad$ Le rendement glotto-articulatoire (/a,s,z/tenus); Rapports a/z et s/z.

- L'intensité moyenne ;

- Le Fondamental Usuel Moyen

Ces paramètres ne permettent pas d'évaluer précisément les erreurs d'articulation spécifiques à la dysarthrie, ou d'autres traits distinctifs tels que le ralentissement du débit, les pauses inappropriées, le voisement ou l'hypernasalisation.

\section{Protocole proposé}

Nous avons proposé un protocole court d'analyse de la parole [Sicard 2019] en ciblant principalement deux paramètres : l'intelligibilité et la fluence. Très inspiré des publications scientifiques sur l'analyse 
de la parole dysarthrique, mais aussi des méta-analyses sur la parole normale, altérée et pathologique pour les enfants, adultes et personnes âgées, nous avons conçu un protocole simple, efficace, totalement informatisé, situant les performances du patient parmi les normes établies et permettant notamment d'évaluer les progrès en lien avec la prise en charge orthophonique. Nous détaillons ciaprès les critères retenus pour évaluer la qualité d'une phrase dans le cadre du bilan, l'analyse de cette phrase, puis l'analyse informatisée de la diadococinésie à l'aide d'indicateurs spécifiques. Enfin, nous explicitons le bilan et l'évaluation de l'intelligibilité et de la fluence. Le protocole se compose de six éléments parmi les incontournables du bilan de la parole :

- Anamnèse

- Autoévaluation

- Enregistrement d'un /a:/ tenu

- Enregistrement de deux phrases

- Analyse des praxies

- Enregistrement de la diadococinésie

\section{Anamnèse}

L'anamnèse proposée regroupe en 8 catégories 200 items possibles, permettant de décrire rapidement les capacités sensorielles, cognitives et de communication du patient, ainsi que des éléments de contexte professionnel. Certains items que la motricité (Figure 9) sont particulièrement pertinents dans le cas de la dysarthrie.

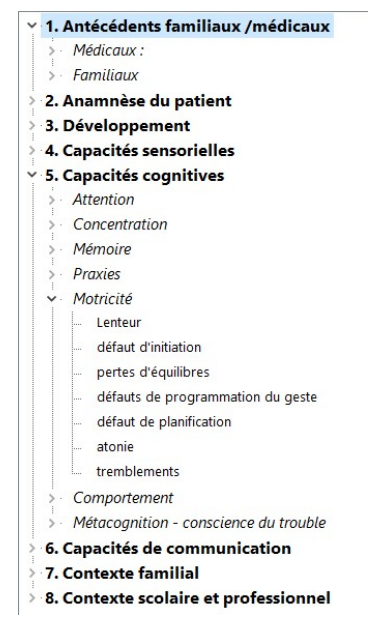

Figure 10 : Eléments d'anamnèse regroupés en 8 catégories

\section{Auto-évaluation}

Différents tests tels que le Speech Handicap Index (SHI) [Rinkel 2008] adapté par [Degroote 2012] sont disponibles pour l'analyse de la parole. Le score du SHI, base sur les réponses à 30 questions, va de 0 à 120 : sujets normaux $<10 / 120$, troubles de la parole : score autour de $50 / 120$, patients dysarthriques autour de 60/120. Le SHI est aussi intégré dans la nouvelle édition de la BECD [Auzou 2019]. Souhaitant réduire significativement le temps de passation tout en gardant la philosophie générale du VHI, nous proposons une auto-évaluation de la parole du patient basée sur seulement 5 questions en lien avec l'intelligibilité, l'expressivité, la fatigabilité, la fluence et l'impact social (Figure 11). Le total est sur 25 points, avec 5 classes proposées : score $<8$ : gêne sévère, $<13$ gêne marquée, $<18$ gêne modérée, et $<23$, gêne discrète, sinon aucune gêne. Le score total est disponible immédiatement en fonction de la place des différents curseurs et le score de gêne est affiché. 

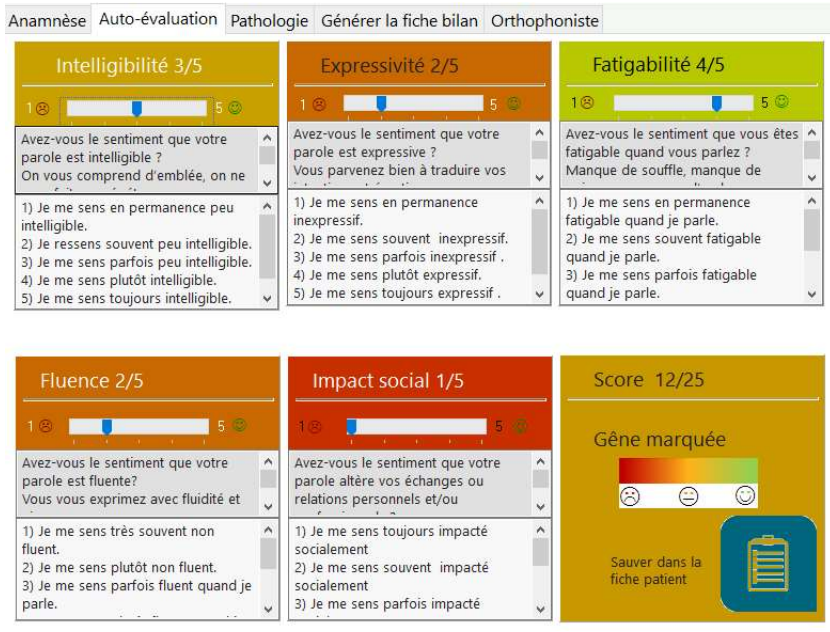

Figure 11 : Auto-évaluation du patient avec 5 questions, score de 1 à 5 , total sur 25

\section{Analyse du /a:/ tenu}

L'enregistrement d'une voyelle /a:/ tenue nous semble important dans le cadre du bilan orthophonique de la dysarthrie pour évaluer les capacités de phonation du patient, la richesse harmonique /nasalisation, le maintien d'un certain niveau de puissance tout au long de la production. Les caractéristiques de phonation sur la voyelle ouverte /a:/ sont une métrique pertinente pour évaluer les progrès du patient en lien avec des rééducations intensives ciblant la puissance de phonation telles que [Fox 2012] ou [Robertson 1984]. Sur la figure 12, on note une durée de phonation effective de 9.9 secondes (Norme 15 secondes pour un homme de 75 ans), et différentes altérations, notamment un rapport bruit/signal élevé et une instabilité en amplitude marquée.

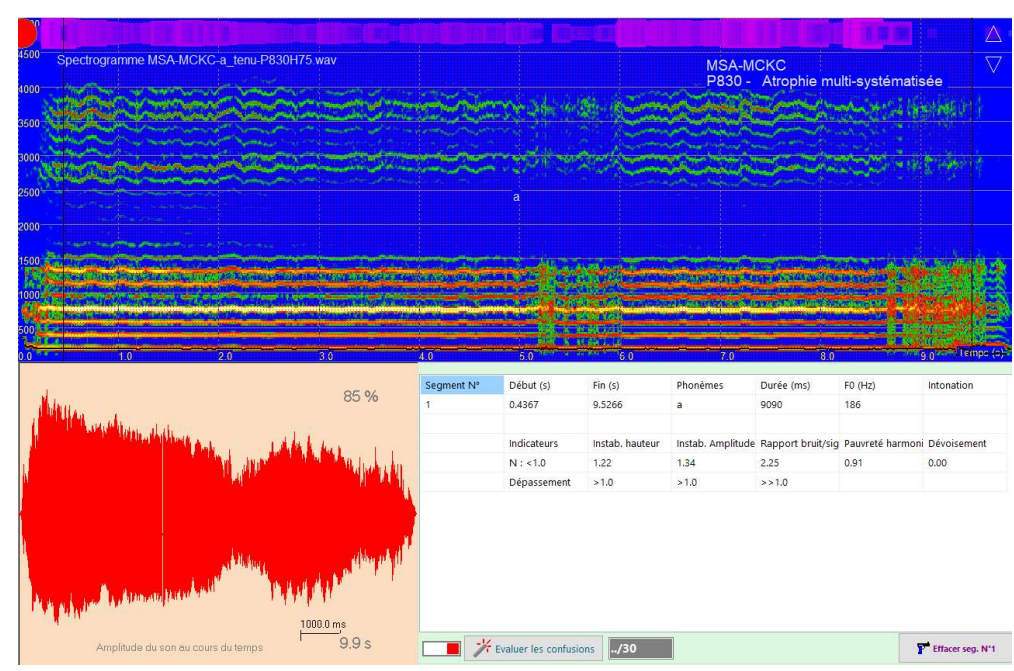

Figure 12 : Enregistrement du /a:/d'un patient MSA : faible durée de phonation, bruit important et instabilité en hauteur et amplitude

\section{Analyse de phrases}

Qualité d'une phrase en bilan orthophonique

Nous avons listé dans [Sicard 2019] un ensemble de critères permettant d'évaluer la pertinence d'une phrase dans le cadre du bilan de la parole :

- Elle est courte

- Elle comprend les extrêmes vocaliques

- Elle comprend les extrêmes consonantiques 
- Elle couvre au mieux les processus de parole

- Elle comprend les phonèmes qui ont le plus fort taux d'occurrence

- Elle est peu redondante

- Elle comporte des accents et contours prosodiques variés

- Sa phonotactique est proche de la langue française.

Une dizaine d'indicateurs telles que "occurrence ", "redondance ", "difficulté ", etc. ont été combinés de manière linéaire ou non linéaire, permettant d'aboutir à des scores de qualité indicatifs, ayant pour finalité de comparer les phrases entre elles pour tenter d'identifier la plus pertinente. Nous avons ainsi obtenu le score le plus élevé sur la phrase "Bonjour Monsieur Tralipau » (Figure 13) que nous proposons dans le cadre du bilan.

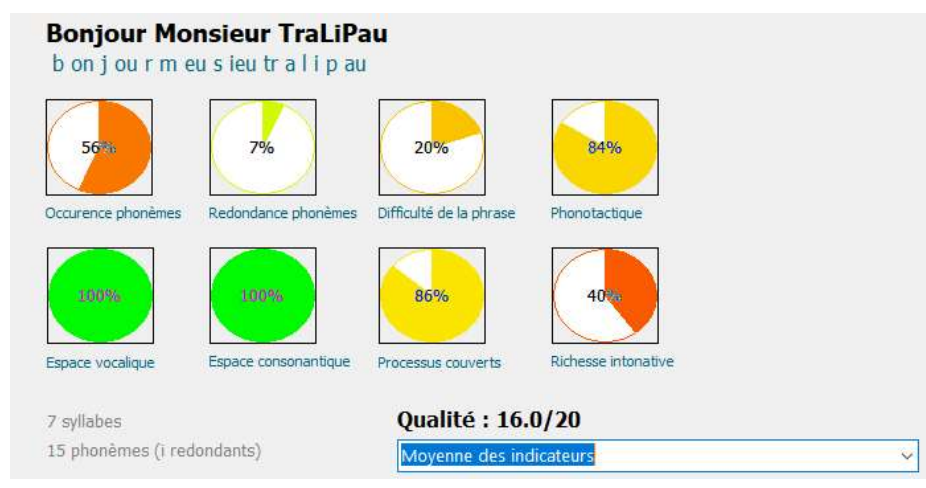

Figure 13 : Evaluation de la qualité de la phrase par défaut «Bonjour Monsieur Tralipau » [Sicard 2019]

\section{Enregistrement de la phrase}

L'enregistrement du patient est effectué en suivant étape par étape et scrupuleusement le protocole décrit dans l'interface utilisateur, en utilisant au préalable les modèles homme, femme et enfant préenregistrés. Une fois l'enregistrement réalisé, la segmentation est effectuée à l'aide du spectrogramme de l'écoute sélective en réajustant les frontières phonémiques grâce à l'allure temporelle. Le clinicien rentre dans le tableau le phonème entendu et le logiciel cote lui-même les altérations.

Différentes informations telles que la constriction, l'explosion et la richesse harmonique facilitent la segmentation. Une fois que tous les phonèmes sont segmentés, il est possible d'évaluer les erreurs, omissions et confusions par comparaison entre la production attendue et la production du patient, ce qui génère un score de phonème correct sur 30, dans le cas de la phrase /Bonjour Monsieur Tralipau/. L'orthophoniste complète des informations supplémentaires concernant le respect de la phrase, l'oubli de mots, la contamination entre phonèmes et d'éventuelles répétitions repérées. Cette analyse acoustique augmente significativement le taux de consensus comparée à l'analyse subjective seule même avec enregistrement. 


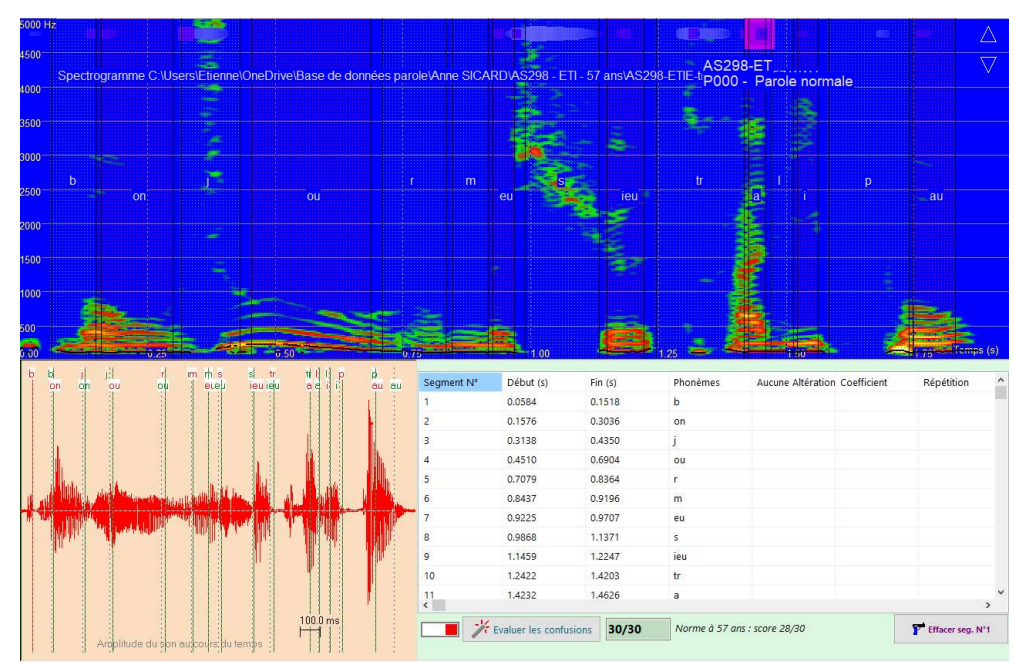

Figure 14 : Segmentation de la phrase « Bonjour Monsieur Tralipau », parole normale (Homme 57 ans)

Analyse de la prosodie

L'analyse de la prosodie de la phrase permet d'évaluer les paramètres suprasegmentaux de la parole tels que l'intonation, la hauteur de la voix, les accents toniques, les pauses inappropriées et le débit de la parole. On trouve une littérature abondante sur l'analyse de la prosodie, notamment dans le contexte de la dysarthrie [Teston 2000]. La segmentation préalable améliore les calculs d'intonation en évitant la détection erronée de F0 dans des portions non voisées.

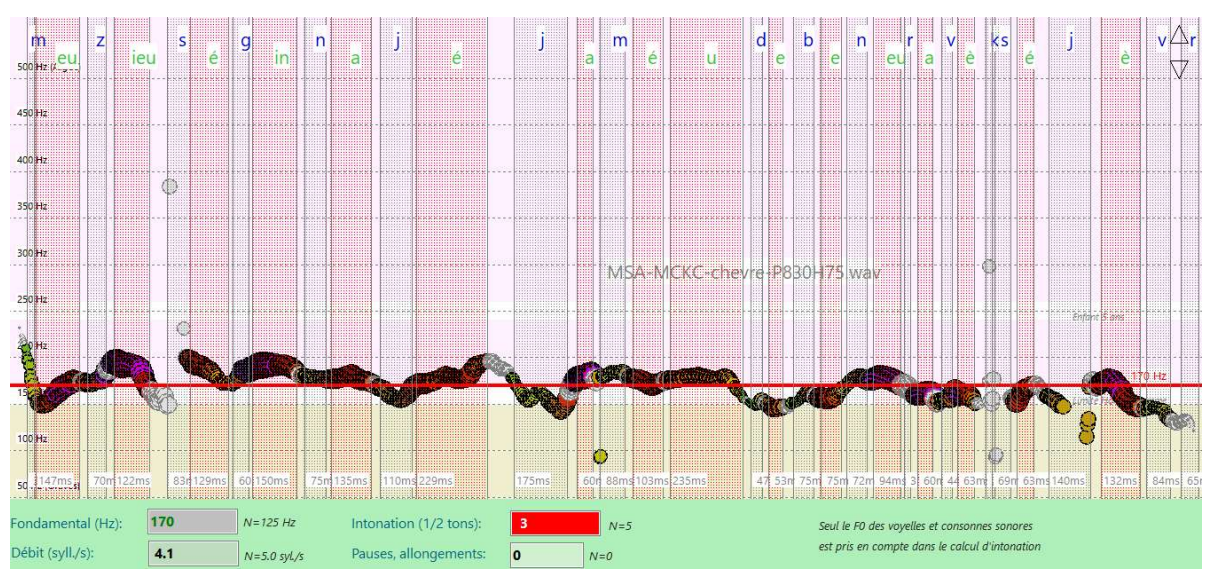

Figure 15 : Evaluation de la prosodie d'un patient dysarthrique (Homme 75 ans)

Les éléments de prosodie tels que le débit des syllabes, I'intonation, les pauses et allongements inappropriés sont ainsi évalués. Dans le cas du patient dysarthrique de la figure 15 (MSA), la parole est monotone, le fondamental variant peu autour de sa valeur moyenne ( $31 / 2$ tons au lieu de 5$)$. On n'observe pas ici de pauses ni d'allongements inappropriés.

\section{Analyses d'autres phrases}

Bien que le bilan court de la parole proposé dans [Sicard 2019] n'intègre qu'une seule et unique phrase, nous pensons que le bilan de la dysarthrie peut inclure une $2^{\text {ème }}$ phrase, complémentaire de /Bonjour Monsieur Tralipau/ avec notamment :

- L'inclusion d'un maximum de phonèmes non couverts par la $1^{\text {ère }}$ phrase

- Un contenu prosodique incluant une question 


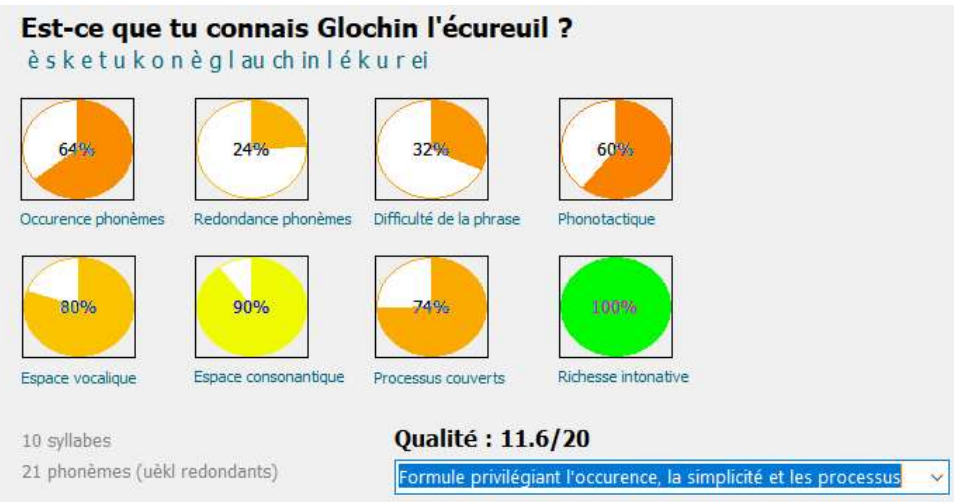

Figure 16 : Evaluation de la qualité de la phrase par défaut « Bonjour Monsieur Tralipau » [Sicard 2019]

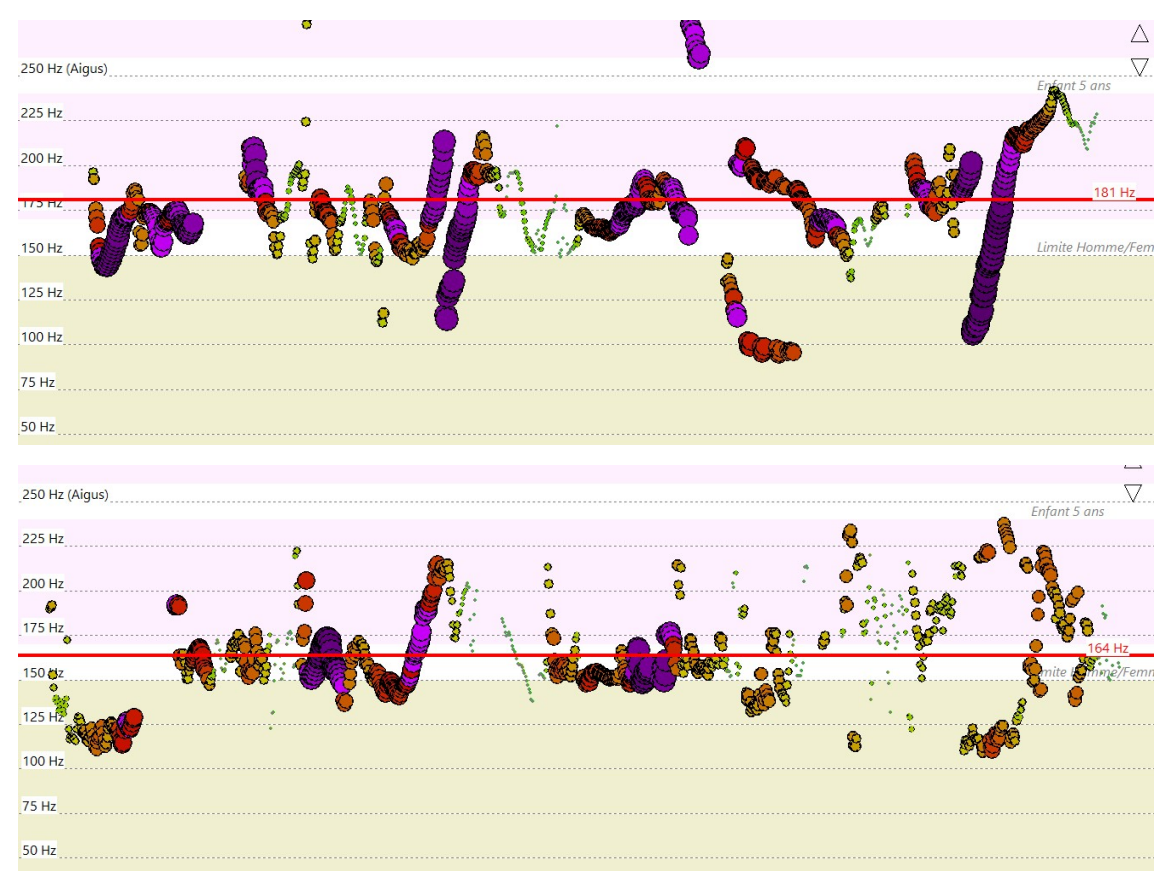

Figure 17 : Contour prosodique de la phrase «Est-ce que tu connais glochin l'écureuil»-Parole normale en haut, parole dysarthrique en bas. Hommes d'âge similaire, reproduisant le modèle pré-enregistré.

Dans le cadre du mémoire d'orthophonie de T. Bardelang [Bardelang 2020], la phrase /Connais-tu Glochin l'écureuil ?/ a été proposée. Celle-ci a une complémentarité élevée par rapport à la phrase par défaut. La différence de contour prosodique, notamment en fin de phrase pour la partie interrogative, est assez nette entre le témoin et le patient dysarthrique (Fig. 17). Elle comporte en outre un logatome de deux syllabes, la formulation usuelle « est-ce que » ainsi qu'un mot faisant partie du vocabulaire de base incluant une triphtongue.

Dans le cadre du projet Voice4PD [Voice4PD] sur le diagnostic différentiel précoce des syndromes Parkinsoniens, nous avons aussi étudié la phrase /Monsieur Seguin n'avait jamais eu de bonheur avec ses chèvres/ extraite d'une nouvelle d'A. Daudet. La qualité de la phrase est principalement pénalisée par la longueur totale,une redondance importante des phonèmes et la couverture partielle du triangle vocalique. 


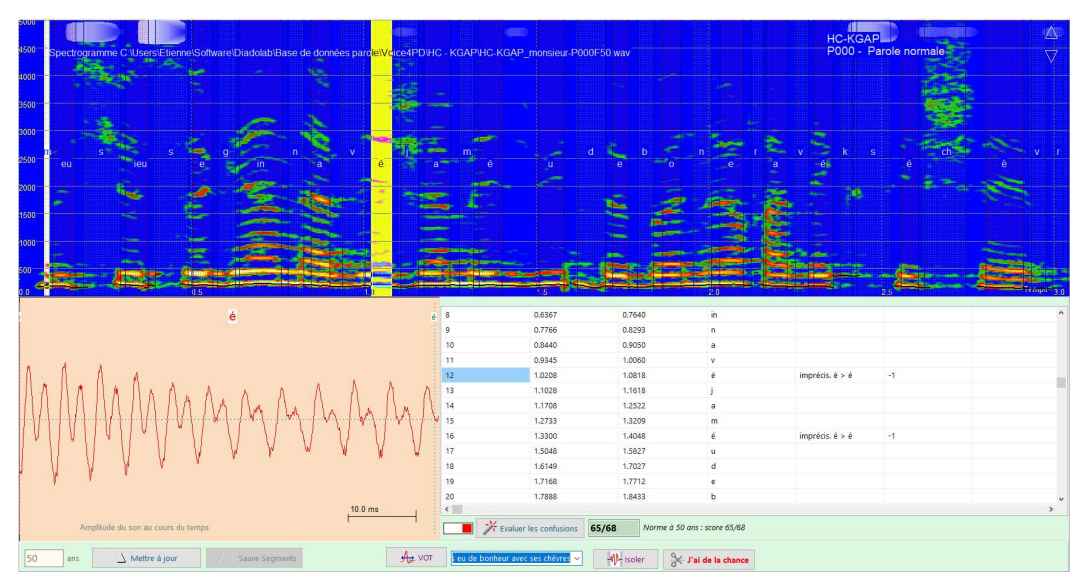

Figure 18 : Segmentation et étiquetage des phonèmes perçus, afin d'établir un score d'articulation

A noter que si l'on cherche à exacerber les erreurs d'articulation spécifiques à la dysarthrie hypokinétique, les occlusives voisées $(/ \mathrm{b} / \mathrm{d} / \mathrm{g} /)$ et non voisées $(\mathrm{p} / \mathrm{t} / \mathrm{k})$ sont à privilégier, ainsi que les voyelles pour lesquelles l'hyper-nasalité est la plus marquée, comme le /i:/ [Novotny 2016].

\section{Enregistrement de la diadococinésie}

Nous avons eu accès à différents corpus de parole normale et pathologique incluant des enregistrements de /PaTaKa/, que nous avons complété par des enregistrements en provenance d'orthophonistes membre du laboratoire LURCO, ERU 46 [Lurco]. Au total, plus de 200 échantillons de parole adulte, normale et pathologique (IPD, MSA \& PSP) ont été utilisés pour l'analyse de la diadococinésie. Une étude bibliographique approfondie, notamment des travaux de [Rusz 2015] a permis de sélectionner quatre indicateurs spécifiques d'altération de la diadococinésie, puis de les valider en retrouvant les principales tendances publiées :

- $\quad$ Atonie des consonnes plosives /p/, /t/, /k/ : voisement, faible explosion

- $\quad$ Débit des syllabes trop lent

- Irrégularité du débit des syllabes

- Instabilité en puissance au cours de la réalisation

Ces indicateurs sont évalués sur 7 secondes de /PaTaKa/ pour les adultes, sans segmentation préalable. Les quatre indicateurs d'altération de la diadococinésie sont normalisés, c'est-à-dire que 1.0 représente la limite au-delà de laquelle l'articulation n'est plus considérée comme normale. Le seuil est ajusté selon l'âge, notamment pour le débit selon la tendance observée figure 8. Dans le cas de la figure 19, nous sommes en présence d'une atonie marquée ainsi qu'une instabilité en puissance. Le débit est limite et la régularité de la production bonne. Nous donnons ci-après quelques précisions sur les métriques analysées par ces quatre indicateurs.

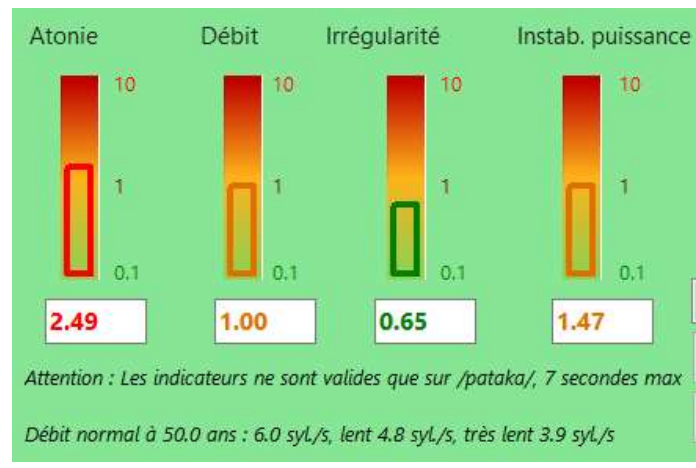

Figure 19: 4 indicateurs d'altération de la diadococinésie implémentés dans DIADOLAB [Menin-Sicard 2019b] 


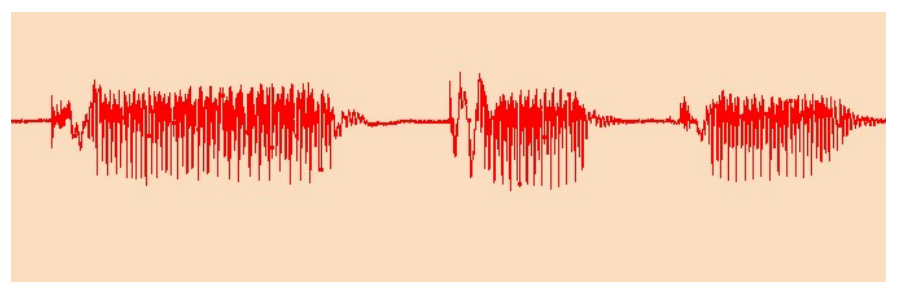

$/ p / t / k /$ correct : atonie faible

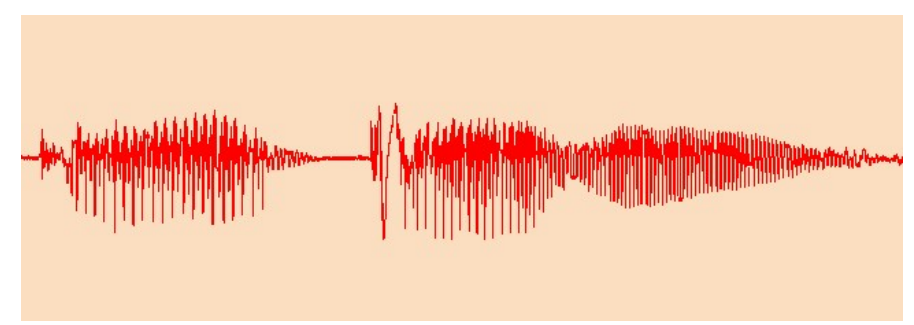

$/ p / t / k /$ incorrect : atonie élevée sur la $3^{\text {ème }}$ syllabe

Figure 20 : la $3^{\text {ème }}$ syllabe est voisée, l'enchainement de la $2^{\text {ème }}$ et $3^{\text {ème }}$ syllabe se fait sans occlusion

\section{Indicateur d'Atonie}

L'indicateur d'atonie sanctionne la faiblesse de l'explosion $d u / p / t / k /$ par rapport à l'énergie de la voyelle /a/. II sanctionne l'absence de consonne ou le voisement de l'explosion. II s'élève aussi en cas de pauses inappropriées entre les syllabes. Dans la Fig. 20 en haut, les plosives sont bien identifiées, l'atonie est donc faible, en dessous de 1.0, tandis que dans la figure du bas, la $3^{\text {ème }}$ plosive disparaît dans le voisement, l'atonie s'élève au-dessus de 1.0. L'indicateur est particulièrement élevé dans les dysarthries spastiques et ataxiques.

\section{Indicateur de débit}

L'indicateur de débit sanctionne un rythme des syllabes plus faible que la norme selon l'âge. II est calculé par Transformée de Fourier du profil de puissance. Pour les adultes, la norme est autour de 6 syllabes/seconde. L'indicateur s'élève si la valeur du débit est plus faible que la norme. L'indicateur s'élève aussi en cas de pauses inappropriées entre les syllabes. Dans le cas de la figure 21, le débit de 4.7 syllabes/secondes est considéré comme lent, l'indicateur correspondant est significativement supérieur à 1.0 .

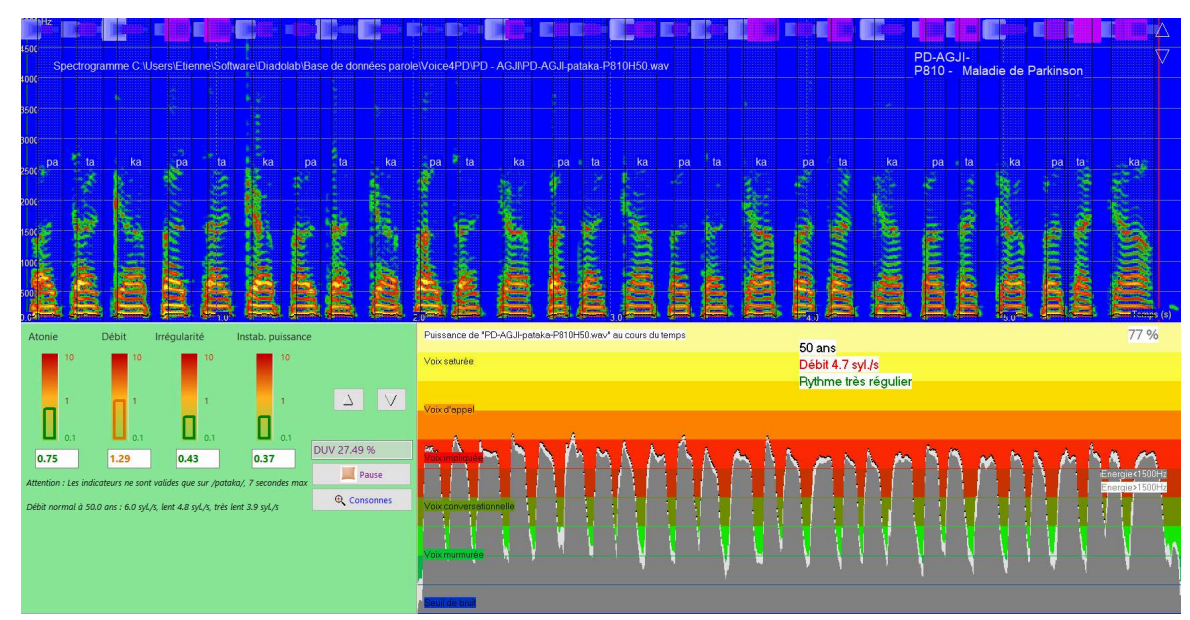

Figure 21 : Débit de diadococinésie inférieur à la norme (Maladie de Parkinson) 


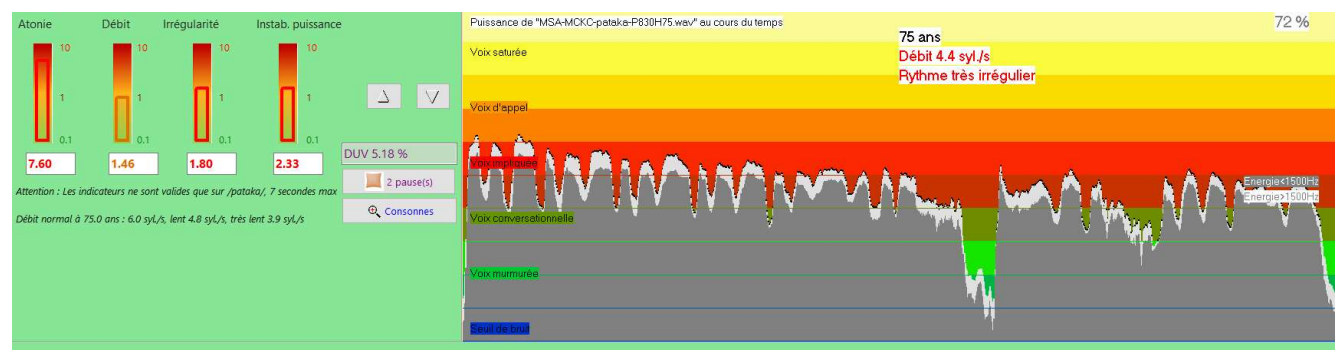

Figure 22: /pataka/ d'un patient atteint de MSA : le débit est lent et le rythme irrégulier. On note aussi une interruption de la diadococinésie

\section{Indicateur d'irrégularité}

L'indicateur d'irrégularité sanctionne des variations de durée des syllabes. Il est calculé d'après la Transformée de Fourier du profil de puissance. Dans la dysarthrie en lien avec MSA et PSP, certaines syllabes sont de durées courtes, d'autres significativement plus longues. Dans le cas de maladie de Parkinson de la figure 21, le rythme est considéré comme très régulier, l'indicateur " Irrégularité » est vert, car très en dessous de 1.0. Par contre, pour le patient MSA de la figure 22, le rythme est considéré comme très irrégulier, à cause de modifications significatives des durées des syllabes. De ce fait, I'indicateur correspondant est significativement au-dessus de 1.0.

\section{Indicateur d'Instabilité en puissance}

L'indicateur d'instabilité en puissance sanctionne des variations de puissance au cours du temps. Dans le cas de dysarthries sévères, la puissance est souvent en décroissance rapide, comme l'illustre la figure 22 pour un patient MSA. L'indicateur « Instab. Puissance » est alors très au-dessus de 1.0.

\section{Analyse des praxies}

Nous disposons d'animations de différentes praxies spécifiques à la parole que nous demandons au patient de reproduire. L'orthophoniste classe les 5 praxies (Figure 23) selon 3 niveaux: bien, partiellement ou mal réalisé. Cela donne un score allant de 5 à 15 points. Ici le patient dysarthrique éprouve beaucoup de difficultés à reproduire correctement et avec l'amplitude désirée les différentes praxies. Le score total est de 8/15.

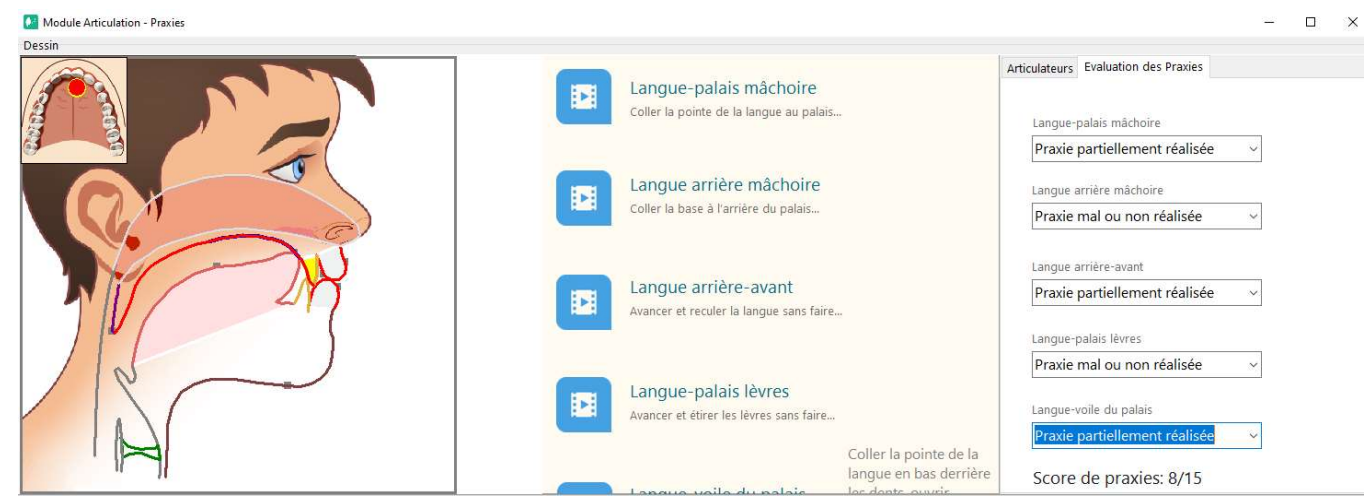

Figure 23 : Evaluation par le thérapeute de la bonne réalisation de 5 praxies

\section{Synthèse du bilan de dysarthrie}

Le bilan de la dysarthrie consiste à exploiter les données en lien avec la voyelle /a:/ tenue, les 2 phrases enregistrées et la diadococinésie, complétés avec des évaluations réalisées par l'orthophoniste, permettant de synthétiser les données grâce à une fiche bilan. La figure 24 dresse le résumé des éléments utilisés et leur exploitation. 


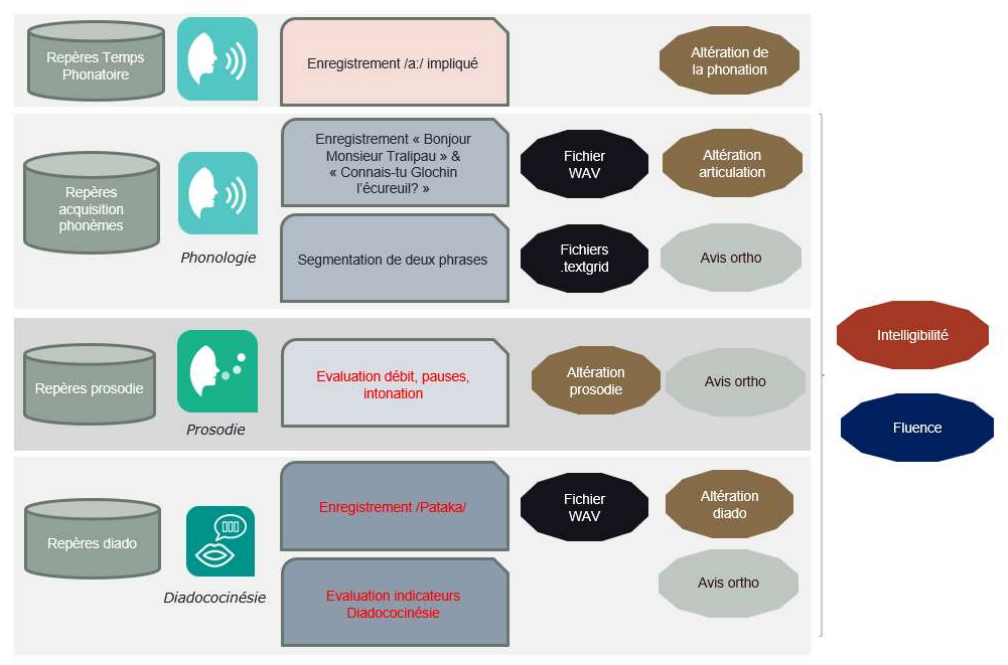

Figure 24 : Evaluation de l'intelligibilité et de la fluence du patient dysarthrique à partir des données calculées sur deux phrases et la diadococinésie

\section{Synthèse : évaluation de l'intelligibilité}

L'intelligibilité est altérée par :

- Un score d'articulation plus bas que la norme : plus il est bas, moins l'intelligibilité est bonne

- Un non-respect de la phrase

- Une contamination élevée (frontières phonémiques floues, difficile à déterminer)

- Une intonation en dessous de la norme

Deux indicateurs anormaux prépositionneront l'intelligibilité à « intelligibilité moyenne ». Quatre indicateurs ou des scores d'articulation très inférieurs à la norme prépositionneront l'intelligibilité à " intelligibilité altérée ". L'orthophoniste peut modifier ou nuancer ces évaluations de synthèse.

Synthèse : évaluation de la fluence

La fluence est altérée par :

- Des répétitions

- Un débit plus lent ou plus rapide que la norme

- Des pauses inappropriées

- Des indicateurs de diadococinésie élevés

- Le non-respect des enchaînements de /PaTaKa/

- Des reprises inspiratoires dans /PaTaKa/

Deux indicateurs anormaux prépositionneront la fluence à " fluence moyenne ». Quatre indicateurs très inférieurs à la norme prépositionneront la fluence à « fluence altérée ». Dans le cas du patient dysarthrique (Figure 25), plusieurs éléments indiquent une altération de la parole :

- Un score d'articulation bas sur la phrase /Bonjour Monsieur Tralipau/

- Une durée de phonation sur le /a:/ de 5.7 secondes seulement (norme à $15 \mathrm{~s}$ )

- Une intonation peu conforme, notamment sur la $2^{\text {ème }}$ phrase (Question).

- Une diadococinésie altérée

- Une pause inappropriée dans la prosodie

- Un score de praxie très bas 


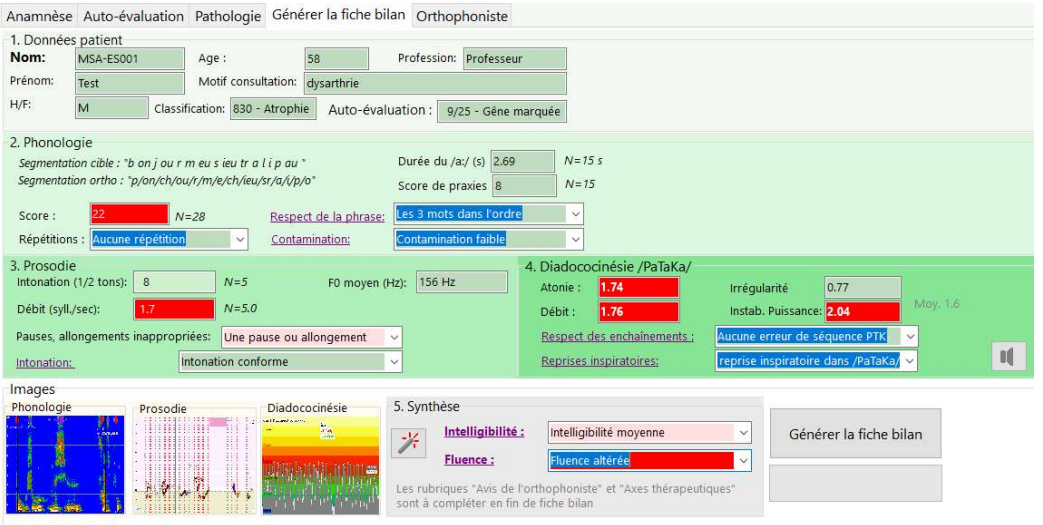

Figure 25 : Synthèse de l'évaluation de l'intelligibilité et de la fluence du patient dysarthrique

\section{Evolution Avant/Après prise en charge orthophonique}

L'intérêt majeur d'un bilan court de la parole est de permettre d'évaluer l'évolution des performances du patient avant et après suivi orthophonique, ou à différents points d'étape, en suivant le même protocole. Nous pouvons alors comparer l'évolution du score d'articulation, de la prosodie et des indicateurs de diadococinésie. Le bilan d'évolution peut ainsi mettre en évidence des progrès, une stagnation ou une régression des performances du patient pris en charge. Dans le cas de la figure 26, on note une amélioration du score d'articulation, même si certaines consonnes demeurent altérées, comme le « $b$ » initial. Un gain de 3 points, notamment dans les fricatives $/ \mathrm{ch} / \mathrm{j} /$ est constaté.

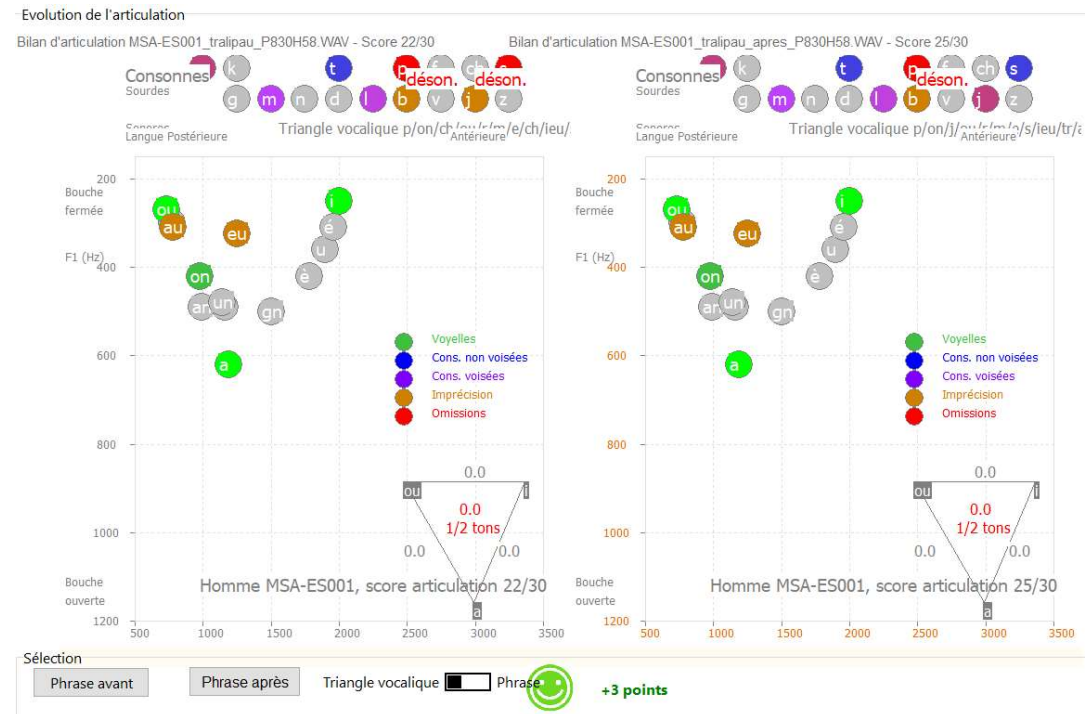

Figure 26 : Evolution du score d'articulation du patient dysarthrique avant/après prise en charge intensive-Gain de 3 points

De plus, les progrès en lien avec une prise en charge intensive telle que LSVT ${ }^{\mathrm{TM}}$ [Fox 2012] ou d'autres approches peuvent être visualisés au cours du suivi, comme illustré Figure 27 sur un /a:/ tenu. On observe principalement un allongement de la durée de phonation, qui passe de pathologique à limite. On note aussi quelques progrès sur la puissance de phonation. 

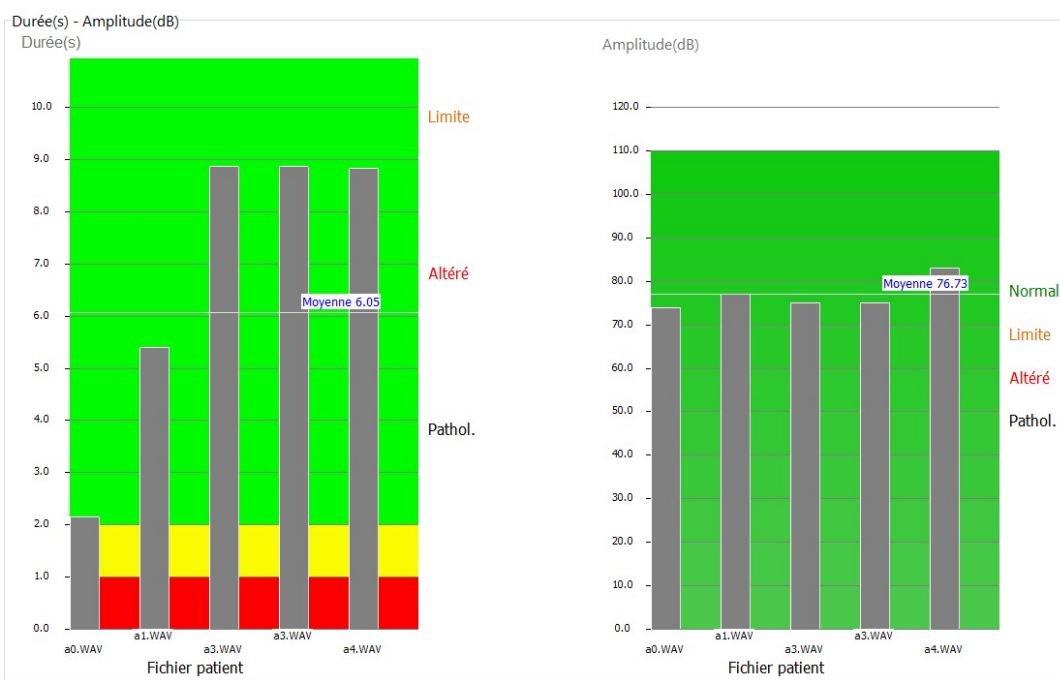

Figure 27 : Evolution de la durée et puissance du /a:/ au cours de la prise en charge orthophonique, patient atteint de la Maladie de Parkinson

\section{Conclusion}

Dans cet article, nous avons décrit une méthodologie de bilan rapide de la dysarthrie et l'atelier logiciel associé permettant d'évaluer rapidement l'intelligibilité et la fluence du patient. Nous avons décrit deux épreuves spécifiques, l'une qui concerne l'évaluation de l'articulation et de la prosodie sur la base d'une courte phrase, l'autre qui se focalise sur l'altération de la diadococinésie sur la base d'une répétition de /PaTaKa/. Nous avons étayé le choix de la phrase, et montré comment la segmentation assistée des phonèmes entendus permettent d'établir un score d'articulation, de même que la prosodie, les pauses inappropriées, et les diverses altérations de la fluence. L'illustration des indicateurs d'altération de la diadococinésie montre des caractéristiques spécifiques des syndromes Parkinsoniens : atonie des plosives, ralentissement du débit, irrégularité du débit, et irrégularité de la puissance. Nous avons décrit les étapes complémentaires du bilan composées de l'auto-évaluation rapide du patient, l'analyse d'une voyelle tenue, l'évaluation des praxies ainsi que l'analyse de contours prosodiques variés. La rapidité du bilan permet de comparer les performances avant/après prise en charge orthophonique, afin d'objectiver les progrès du patient.

\section{Références}

1. Ackermann, H., Hertrich, I., \& Hehr, T. (1995). Oral diadochokinesis in neurological dysarthrias. Folia phoniatrica et logopaedica, 47(1), 15-23.

2. Antolík T.K., Fougeron C., (2013) Consonant distortions in dysarthria due to Parkinson's disease, Amyotrophic Lateral Sclerosis and Cerebellar Ataxia, Interspeech 2013

3. Auzou, P., \& Rolland-Monnoury, V. (2006). BECD: batterie d'évaluation clinique de la dysarthrie. Ortho édition.

4. Auzou, P. (2009). Définition et classifications des dysarthries. Rééducation orthophonique, 47(239), 31-42.

5. Auzou, P. (2000), Voice onset time in aphasia, apraxia of speech and dysarthria: a review, clinical linguistics \& phonetics, 2000 , vol. 14, no. 2, 131-150

6. Bardelang, T. (2020) L'apport d'une phrase supplémentaire au protocole de DIADOLAB3, Mémoire d'orthophonie de l'Université de Lyon, en cours. 
7. Blanc, E., Giusti, L. (2014). Evaluation de l'intelligibilité de la parole dans les dysarthries: adaptation en français de la version révisée du Frenchay Dysarthria Assessment [FDA-2], Certificat d'orthophonie, Marseille

8. Darley FL, Aronson AE, Brown JR. Clusters of deviant speech dimensions in the dysarthrias. J Speech Hear Res 1969, 12 : 462-496.

9. Degroote, G., Simon, J., Borel, S., \& Crevier-Buchman, L. (2012). The French version of Speech Handicap Index: validation and comparison with the voice handicap index. Folia Phoniatrica et Logopaedica, 64(1), 20-25.

10. Duffy, J. R. (2013). Motor Speech Disorders-E-Book: Substrates, Differential Diagnosis, and Management. Elsevier Health Sciences.

11. Duez, D. (2006). Consonant and Vowel Duration in Parkinsonian French Speech. 2006, University of Dresden, pp.101-105, 2006. <hal-00136745>

12. Enderby, P. M., \& Palmer, R. (2008). Frenchay dysarthria assessment. Pro-ed.

13. Fonville, S., (2008). Accuracy and inter-observer variation in the classification of dysarthria from speech recordings. Journal of Neurology, 255(10), 1545-1548

14. Fougeron C., et al. (2016) MonPaGe : un protocole informatisé d'évaluation de la parole pathologique en langue française. Actes du colloque UNADREO «Orthophonie et technologies innovantes ", Dec. 2016

15. Fox, C., Ebersbach, G., Ramig, L., \& Sapir, S. (2012). LSVT LOUD and LSVT BIG: behavioral treatment programs for speech and body movement in Parkinson disease. Parkinson's Disease, 2012.

16. Ghio, A. et al. (2016). Quels tests d'intelligibilité pour évaluer les troubles de production de la parole?. Journées d'Etude sur la Parole, 2016, Paris, France. pp.589-596. hal-01372037

17. Jan, M. (2007) " Evaluation instrumentale de la dysarthrie en France ", in " Les dysarthries », pp 119-122, CD d'accompagnement pp 259-269, Auzou, P., Rolland Monnoury, V., Pinto, S., \& Öszancak, C.

18. Kent, R. D., Weismer, G., Kent, J. F., \& Rosenbek, J. C. (1989). Toward phonetic intelligibility testing in dysarthria. Journal of Speech and Hearing Disorders, 54(4), 482-499.

19. Locco, Julie. La production des occlusives dans la maladie de Parkinson. 2005. Thèse de doctorat. Université de Provence. Faculté des lettres et sciences humaines.

20. Laboratoire Unadreo de Recherches Clinique Orthophonique (LURCO) http://lurco.unadreo.org

21. Martinez-Sanchez, F. Estudio controlado del ritmo del habla en la enfermedad de Parkinson, Neurología, Volume 31, Issue 7, September 2016, Pages 466-472

22. Menin-Sicard, A., \& Sicard, E. (2016). Evaluation et réhabilitation de la voix: Approche clinique et objective. De Boeck Supérieur.

23. Menin-Sicard, A., Sicard, E. (2019) DIADOLAB 3 - Logiciel d'évaluation et de rééducation de la parole - Manuel d'utilisation. Archives ouvertes du CNRS https://hal.archives-ouvertes.fr/hal$\underline{02073314}$

24. Menin Sicard, A., Sicard, E. (2019). Méthodologie d'évaluation objective de la phonologie, de la fluence et de la prosodie : vers un bilan rapide à destination des orthophonistes. Journées de Phonétique Clinique, Mons, Belgique, Mai 2019. hal-02127039

25. Miller N., (2017). Utility and accuracy of perceptual voice and speech distinctions in the diagnosis of Parkinson's disease, PSP and MSA-P. Neurodegener Dis Manag. 2017 Jun;7(3):191-203.

26. Movement Disorder Society Task Force on Rating Scales for Parkinson's Disease. (2003). The Unified Parkinson's Disease Rating Scale (UPDRS): status and recommendations. Movement disorders: official journal of the Movement Disorder Society, 18(7), 738. 
27. Novotny, M., Rusz, J., Spalenka, K., Klempir, J., Horakova, D., \& Ruzicka, E. (2017). Acoustic Evaluation of Nasality in Cerebellar Syndromes. In INTERSPEECH (pp. 3132-3136).

28. Orozco-Arroyave, J. R. (2014). Phonation and articulation analysis of Spanish vowels for automatic detection of Parkinson's disease. Text, Speech and Dialogue (TSD 2014), Proceedings; Springer, 2014, pp. 374-381

29. Payan, C. A., et al. (2011). Disease severity and progression in progressive supranuclear palsy and multiple system atrophy: validation of the NNIPPS-Parkinson Plus Scale. PLoS One, 6(8), e22293.

30. Pierce, J.E. (2013) Alternating and sequential motion rates in older adults, International Journal of Language \& Communication Disorders · May 2013

31. Pinto, S., et. Al. (2004). Treatments for dysarthria in Parkinson's disease. The Lancet Neurology, 3(9), 547-556.

32. Pinto, S., Ghio, A., Teston, B., \& Viallet, F. (2010). La dysarthrie au cours de la maladie de Parkinson. Histoire naturelle de ses composantes: dysphonie, dysprosodie et dysarthrie. revue neurologique, 166(10), 800-810.

33. Roland, V. (2017). Dynamique phonétique et contrôle moteur dans la maladie de Parkinson: analyse du contrôle de la production des glides. JPC 2017 Paris

34. Robertson, S. J., \& Thomson, F. (1984). Speech therapy in Parkinson's disease: a study of the efficacy and long term effects of intensive treatment. British Journal of Disorders of Communication, 19(3), 213-224.

35. Rinkel, R., et al. (2008). Speech Handicap Index in patients with oral and pharyngeal cancer: Better understanding of patients' complaints. Head \& Neck. 30. 868 - 874.

36. Rusz, Jan, Speech disorders reflect differing pathophysiology in Parkinson's disease, progressive supranuclear palsy and multiple system atrophy, Journal of Neurology. 2015; 262(4): 992

37. Rusz, J., (2011). Quantitative acoustic measurements for characterization of speech and voice disorders in early untreated Parkinson's disease. The journal of the Acoustical Society of America, 129(1), 350-367.

38. Sarr, M.M., (2013) comprendre et évaluer la dysarthrie parkinsonienne : revue de la littérature. Rev Int Sc Méd 2013;15,3:184-190.

39. Shriberg, L. D., Kent, R. D., \& Munson, B. (1995). Clinical phonetics. Boston: Allyn \& Bacon.

40. Sicard, E., Menin-Sicard, A. (2019) Analyse de la qualité des phrases pour un bilan objectif de la parole, Archives ouvertes du CNRS, https://hal.archives-ouvertes.fr/hal-02389764

41. Sicard, E., Perrière, S., Menin-Sicard A. (2013)."Développement de nouveaux outils de mesures de la qualité de la voix en vue d'une implémentation dans le logiciel VOCALAB" Glossa 113, pp. 63-80, Archives ouvertes du CNRS, hal-00977731

42. Shriberg, L. D., (2010). Extensions to the speech disorders classification system (SDCS). Clinical linguistics \& phonetics, 24(10), 795-824.

43. Teston, B. (2000) L'évaluation objective des dysfonctionnements de la voix et de la parole; 1ère partie: les dysarthries. Travaux Interdisciplinaires du Laboratoire Parole et Langage d'Aix-en-Provence (TIPA), Laboratoire Parole et Langage, 2000, 19, pp.115-154. hal-00173551

44. Tranchant C. Autres syndromes parkinsoniens. Presse Med. (2017),

45. Tykalova, T., Rusz, J., Klempir, J., Cmejla, R., \& Ruzicka, E. (2017). Distinct patterns of imprecise consonant articulation among Parkinson's disease, progressive supranuclear palsy and multiple system atrophy. Brain and language, 165, 1-9.

46. Voice4PD : https://project.inria.fr/voice4pdmsa/ 


\section{Glossaire}

- AD Ataxic Dysarthria

- AMR : Alternating motion rate

- AMS : Atrophie Multi-Systématisée

- ALS: Amyotrophic Lateral Sclerosis

- AOS: Apraxia of Speech

- APS: Atypical Parkinsonian Syndromes

- CA: Cerebellar Ataxia

- CD : Choreatic Dysarthria

- CV : Consonne Voyelle

- CCV : Consonne consonne voyelle

- DYS: Dysarthria

- DD : dystonic Dysarthria

- DDK : Diadochokinetic

- FD : Flaccid Dysarthria

- $\mathrm{HC}$ : Healthy control

- HOD : hypokinetic Dysarthria

- HYD : Hyperkinetic Dysarthria

- HD : Huntington's disease

- IPD: Idiopathic Parkinson's disease

- LURCO : Laboratoire Unadreo de Recherches Clinique Orthophonique

- MD : Mixed Dysarthria

- MSA : Multiple system atrophy

- MSA-P : Multiple system atrophy dominant parkinsonism

- MSA-C : Multiple system atrophy dominant cerebellar ataxia

- MSD : Motor Speech Disorders

- NOS: Not otherwise specified

- PD : Parkinson's Disease

- PSP: progressive supranuclear palsy

- SP : Syndromes Parkinsoniens

- $\quad$ SD : spastic Dysarthria

- SDCS : speech disorders classification system

- SMR : Sequential motion rate

- SP : syndromes Parkinsoniens

- $\mathrm{SHI}$ : Speech Handicap Index

- UPDRS : Unified Parkinson's Disease Rating Scale

- VOT : Voice Onset Time

- VHI : Voice Handicap Index 